a familia Acanthaceae incluye alre-

_dedor de 4,000 especies con distribución principalmente en los trópicos y subtrópicos. Cerca de 12 géneros, en los que se incluyen Justicia y Ruellia, tienen distribución pantropical. Alrededor de 350 especies, en 30 géneros, se presentan en México (Daniel, 1995). Ruellia es un género con alrededor de 250-300 especies, que lo hace el segundo más abundante dentro de la familia, y se ha considerado que México es el centro de diversificación de éste, pues alberga al menos 65-70 de las especies conocidas, que lo hace también el segundo género más abundante para el país (Daniel, 1995, 2008; Tripp, 2010). Estudios moleculares (Tripp, 2007) señalan que Ruellia s. 1. es un grupo monofilético y que su polen de tipo esférico, triporado, con escultura reticulada es quizá el mejor carácter que unifica al género.

El género ha sido dividido en 10 a 11 secciones. Una de éstas, la sección Chiropterophila, establecida por Ramamoorthy (1991), ha sido ampliamente estudiada por Tripp (2010). La sección está conformada por 11 especies y se caracteriza por incluir plantas con tallos angulosos y pubescencia glandular, presentar flores en dicasios simples, axilares o terminales, o bien, dicasios compuestos, o flores solitarias, el cáliz profundamente lobado y persistente, las corolas van de amarillas a amarillo pálido, o bien, blancas, con formas urceoladas a funeliformes o funeliformes-dobladas a gibosas, los estambres pueden estar inclusos o exertos, el estilo es piloso y el estigma presenta dos lóbulos filamentosos, en algunos casos uno de ellos más corto.

Las especies de esta sección se presentan preferentemente en zonas montañosas entre los 600 y 2,500 m en bosques de encino, tropical caducifolio, mesófilo de montaña y matorral, y muchas especies tienen afinidad por suelos calizos. La sección es endémica

\title{
Presencia de Ruellia jaliscana Standl. (Acanthaceae. SeCt. Chiropterophila) EN LA FLORA DEL ESTADO DE GUERRERO, MÉXICO.
}

\author{
Ramiro Cruz Durán y yaría Elena García Granados. \\ Departamento de Biología Comparada, Facultad de Ciencias, \\ Universidad Nacional Autónoma de México, México, D.F. \\ 1Autor para la correspondencia: ramcrudur@yahoo.com
}

a México y sus representantes pueden encontrarse en el Faja Volcánica Transmexicana, Sierra Madre del Sur y partes de la Sierra Madre Occidental y Oriental. Estas regiones comprenden los estados de Hidalgo, parte de Veracruz, Puebla, Morelos, suroeste de Oaxaca, oeste de Guerrero hasta Michoacán y Jalisco, suroeste de Nayarit y Zacatecas (Tripp, 2010).

En algunos estudios de la flora para el estado de Guerrero, como los de Jiménez-Ramírez et al. (2003) y Valencia-Ávalos et al. (2011), Acanthaceae figura entre las familias más diversas, con 30 y 24 especies, respectivamente. Asimismo, en estudios de la familia Acanthaceae para el estado de Guerrero (González, 1992) se cuantifican 23 géneros y 73 especies, donde los géneros Ruellia y Justicia son los más abundantes, pues registran 15 y 12 especies, respectivamente. Se menciona también que en el estado Ruellia se le localiza en bosque tropical caducifolio, tropical subcaducifolio y de Quercus.

Según Tripp (2010), los datos de campo y ejemplares de herbario revelan que las recolectas de las especies de la sección Chiropterophila son escasas. De esta forma, algunas especies como Ruellia cedilloi Ramamoorthy o $R$. guerrerensis T.F.Daniel, son conocidas sólo del tipo, o bien de una única colección. Otras son endémicas de un estado, por ejemplo $R$. conzattii Standl. para Oaxaca y $R$. jaliscana Standl. para Jalisco. Sin embargo, en las exploraciones realizadas en la Sierra de Guerrero por el Herbario de la Facultad de Ciencias (FCME), UNAM, se localizaron ejemplares de la familia Acanthaceae, que luego de seguir las claves de Daniel (1995, 1999), Daniel y Acosta-Castellanos (2003), González (1992) y Gibson (1974), se llegó a la conclusión de que correspondían al género Ruellia, ya que presentan hojas opuestas, cuatro estambres fértiles, anteras bitecas múticas, cáliz con cinco lóbulos, corola actinomórfica salveiforme y flores cimosas. Asimismo, después de consultar los trabajos de Ramamoorthy (1991) y Tripp (2010), y de cotejar con los ejemplares depositados en el Herbario Nacional (MEXU), se determinó que estas recolectas corresponden a Ruellia jaliscana Standl., por presentar inflorescencias profusamente ramificadas en panículas de cimas terminales, hojas elípticas de más de $14 \mathrm{~cm}$ de largo y corolas funeliformes. De acuerdo a Tripp (2010) la 


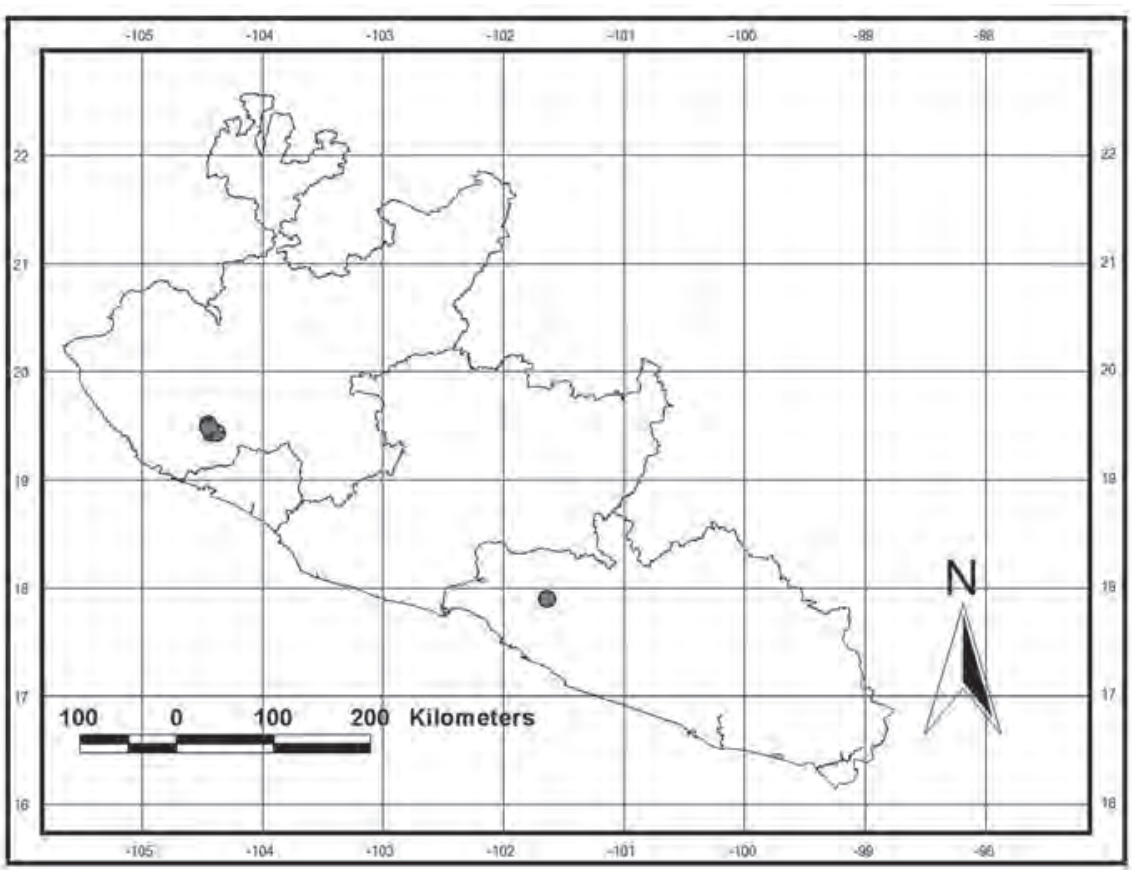

Fig. 1. Distribución conocida de Ruellia jaliscana Standl. en los estados de Guerrero y Jalisco.

especie es común en la Sierra de Manantlán, Talpa de Allende y sus cercanías, en el estado de Jalisco.

Ruellia jaliscana se colectó en el ejido el Aguacate, municipio de Coahuayutla (Figura 1), este sitio se ubica entre $18^{\circ} 12^{\prime} 5.9^{\prime \prime} \mathrm{N}, 101^{\circ} 26^{\prime}$ $8.8^{\prime \prime} \mathrm{O}$, a una altitud de $1,550 \mathrm{~m}$. En la zona, $R$. jaliscana se presenta como un sufrútice de hasta $1.6 \mathrm{~m}$ de alto, en suelos calizos, en bosque de PinusQuercus, con floración en marzo. Este sufrútice frecuentemente está asociado a Ageratum corymbosum Zucc., Eryngium longifolium Cav., Ipomoea lutea Hemsl., Lopezia miniata Lag., Phytolacca icosandra L., Piqueria trinervia Cav., Russelia sarmentosa Jacq. $\mathrm{y}$ Vigna adenantha (G.Mey.) Maréchal, Mascherpa \& Stainier. Presenta hojas de (11.5-) 12-15 (-17) × 5-7 (-9) cm, con hasta 13 pares de venas secundarias, la láminas son elípticas a ligeramente ovadas, con ápice agudo, base decurrente y margen denticulado, el haz es blanco pubescente y con abundantes cistolitos; el envés es seríceo. Las inflorescencias se presentan en panículas de dicasios terminales, los pedúnculos son glandular pubescentes de
2-3 cm de largo, las brácteas son filiformes, glandular pubescentes de 4.5$6 \mathrm{~cm}$ de largo, los pedicelos 1-1.5 cm de largo, glandular pubescentes; las flores son amarillo-pálido (de acuerdo al ejemplar de herbario, sin embargo, la etiqueta del ejemplar dice que son blancas), 6.5-7 (-8) cm de largo, con lóbulos del cáliz 4-4.2 ×0.3 cm, glandular pubescentes, la corola funeliforme, con tubo hasta $5 \mathrm{~cm}$ de largo, con parte no expandida de $2.5-3 \mathrm{~cm}$ y parte expandida de $1.8-2 \mathrm{~cm}$, ligeramente pubescente, con los lóbulos $1.7-2 \times 1.3 \mathrm{~cm}$, las anteras de $1 \mathrm{~cm} \mathrm{de}$ largo, múticas, el ovario glabro hasta $6 \mathrm{~mm}$ de largo, el estilo piloso, hasta $3.7 \mathrm{~cm}$ de largo, ligeramente exserto de la corola (Figura 2).

La distribución disyunta de Ruellia jaliscana en el estado de Guerrero y en el estado de Jalisco concuerda con el registro de Acer saccharum Marshall subsp. skutchii (Rehder) A.E. Murray recolectado también en Guerrero, en el municipio de Coahuayutla (Mayorga 1519, FCME, MEXU, IEB), y mencionado por Vargas-Rodríguez (2011) donde se señala que la especie era sólo conocida de la Sierra de Manantlán y Talpa de Allende en el estado de Jalisco. Asimismo, la reciente publicación de Lepidagathis danielli R.Cruz y J.Jiménez-Ram. (Cruz-Durán y Jiménez-Ramírez, 2011b) y Holographis lizethae R.Cruz y J.JiménezRam. (Cruz-Durán y Jiménez-Ramírez, 2011a) en el estado de Guerrero es un indicativo que en el estado todavía queda mucho por conocer para la familia Acanthaceae.

Ejemplares examinados: México. Guerrero. Mpio. Coahuayutla de José María Izazaga. El Aguacate, $4.2 \mathrm{~km}$ al NE, $18^{\circ} 12^{\prime} 5.9^{\prime \prime}, 101^{\circ} 26^{\prime} 8.8^{\prime}, 1,550 \mathrm{~m}$, bosque de Pinus-Quercus, 24 ene 1999, R. Mayorga S. s.n. (FCME). La escasez de material de herbario de esta especie obedece a que no se ha podido visitar la zona de nuevo, debido, en gran medida, a la inseguridad que prevalece en la región.

\section{Agradecimientos}

Las recolectas de herbario se realizaron con el proyecto R177, Base de Datos de las Regiones prioritarias 113 y 112 en los municipios de Zirándaro y Coahuayutla, Guerrero, financiado por la CONABIO. Susana Valencia Avalos hizo importantes sugerencias al manuscrito. Yareli Joselín Cadena elaboró el mapa de distribución de la especie.

\section{Literatura citada}

Cruz-Durán R. y Jiménez-Ramírez J. 2011a. Holographis lizethae (Acanthaceae), una especie nueva de Guerrero, México. Brittonia 63:321-324.

Cruz-Durán R. y Jiménez-Ramírez J. 2011b. Lepidagathis danielii (Acanthaceae), una especie nueva de la cuenca del Río Balsas en el estado de Guerrero, México. Brittonia 63:334-337.

Daniel T.F. 1995. Flora of Chiapas, Part 4: Acanthaceae. California Academy of Sciences, San Francisco.

Daniel T.F. 1999. Acanthaceae. En: Dávila A.P., Villaseñor R.J.L., Medina L.R. y Téllez V.O. Eds. Flora del Valle de Tehuacán-Cuicatlán. Fascículo 23. Instituto de Biología, Universidad Nacional Autónoma de México, México, D.F. 


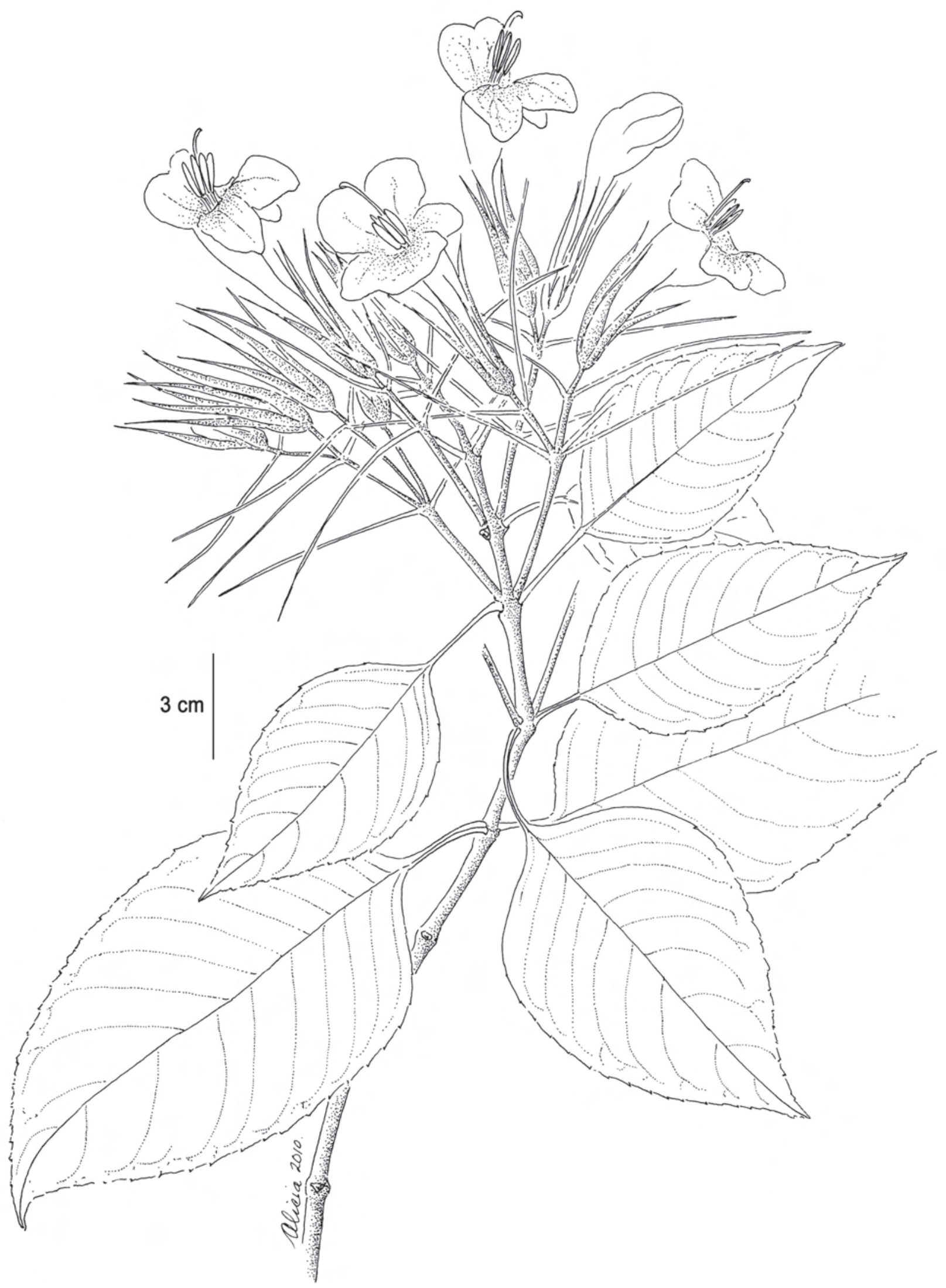

Fig. 2. Rama con flores de Ruellia jaliscana Standl (Mayorga s.n., FCME). 
Daniel T.F. 2008. A new Ruellia (Acanthaceae) from Guerrero, Mexico. Proceedings of the California Academy of Sciences 59:109112.

Daniel T.F. y Acosta-Castellanos S. 2003. Acanthaceae. Flora del Bajio y de Regiones Adyacentes. Fascículo 117. Instituto de Ecología A.C./Consejo Nacional de Ciencia y Tecnología/Comisión Nacional para el Conocimiento y Uso de la Biodiversidad, Pátzcuaro.

Gibson D.N. 1974. Acanthaceae. Acathus Family. En: Standley P.C., Williams L.O. y Gibson D.N. Eds., Flora of Guatemala Part X, pp. 328-461. Field Museum of Natural History, Chicago.

González R.E. 1992. Estudio genérico de la familia Acanthaceae en el estado de
Guerrero. Tesis de Licenciatura. Facultad de Ciencias, Universidad Nacional Autónoma de México. México, D.F. 66 pp.

Jiménez-Ramírez J., Martínez-Gordillo M., Valencia-Ávalos S., Cruz-Durán R., Contreras-Jiménez J.L., MorenoGutiérrez E. y Calónico-Soto J. 2003. Estudio florístico del municipio Eduardo Neri, Guerrero. Anales del Instituto de Biología, Universidad Nacional Autónoma de México, Serie Botánica 74:79-142.

Ramamoorthy T.P. 1991. Ruellia section Chiropterophila (Acanthaceae): a novelty from México. Botanical Journal of the Linnean Society 107:79-88.

Tripp E.A. 2007. Evolutionary relations- hips within the species-rich genus Ruellia (Acanthaceae). Systematic Botany 32:628-649.

Tripp E.A. 2010.Taxonomic revision of Ruellia section Chiropterophila (Acanthaceae): a lineage of rare and endemic species from Mexico. Systematic Botany 35:629-661.

Valencia-Ávalos S., Cruz-Durán R., Martínez-Gordillo M. y Jiménez-Ramírez J. 2011. La flora del municipio de Atenango del Río, estado de Guerrero, México. Polibotánica 32:9-39.

Vargas-Rodríguez Y.L. 2011. Una población relicta de Acer saccharum subsp. skutchii (Aceroideae) en el Estado de Guerrero, México. Acta Botanica Mexicana 95:11-28.

Recibido: 23 de noviembre de 2011

Aceptado: 29 de marzo de 2012 